\title{
The engagement of ALICE with the public during CERN Open Days 2019
}

\section{Despina Hatzifotiadou ${ }^{a, *}$}

${ }^{a}$ INFN Bologna, Italy and EP Department, CERN

E-mail: despina.hatzifotiadou@cern.ch

The ALICE Collaboration uses a multitude of methods to reach out to the public. Visits to the experiment, both underground during LHC shutdown and to the new ALICE exhibition, are among the most important outreach activities. During the CERN Open Days in September 2019, 4600 people visited the ALICE cavern and part of the LHC tunnel; in addition, a variety of activities were organised at LHC Point 2, bringing to children and adults the excitement of science. Talks, simple physics experiments and hands-on activities, involving building the ALICE detector out of cardboard or lego blocks, the cosmic piano and many others kept the whole family entertained. A team of few ALICE members, in collaboration with the CERN central organisers, prepared the event and trained more than 200 enthusiastic volunteers. An overview of this memorable weekend is presented here.

40th International Conference on High Energy physics - ICHEP2020

July 28 - August 6, 2020

Prague, Czech Republic (virtual meeting)

${ }^{1}$ on behalf of the ALICE collaboration

* Speaker 


\section{Introduction}

The CERN Open Days, during the weekend 14-15 September 2019, were a major outreach event, for CERN and for our community. Out of the many visits sites, the LHC experiments had a special appeal, since they were all offering underground visits, which is what really fascinates the public. However, visits to the cavern were only one of the many options on offer. Similarly to the other LHC experiments, and also to what was done on previous occasions (CERN Open Days 2013), at LHC-Point 2, the site of the ALICE experiment, talks, demonstrations and hands-on activities were organised, aiming to arouse the interest of adults and children, inform and entertain them. In the following sections we present briefly what happened during that memorable weekend.

\section{Preparation of the event}

Many months before the event we created a small team consisting of the ALICE GLIMOS (Group Leader In Matters Of Safety) and members of the technical coordination, the outreach coordination, the secretariat and the management, to organise all aspects of Open Days at Point 2. These involved all kinds of logistics (allocation of spaces to activities, preparation of infrastructure), recruiting and training of volunteers and production of outreach material for the occasion. We had regular meetings and also participated in the meetings organised by the CERN core team and the more specific ones for the coordination of the LHC points.

A number of posters presenting the different components of ALICE were designed for the open days, suitable for the general public and available for other outreach events [1].

\section{Visits to the LHC tunnel, the ALICE cavern and the exhibition}

Similarly to Open Days 2013, the underground visit at Point 2 combined part of the LHC tunnel and the ALICE cavern. This time we had the special luck to be allowed to do a relatively long circuit inside the service tunnel and the main tunnel. In this way the visitors could get a complete picture of both accelerator and experiment. For many guides also it was a unique opportunity to see the LHC tunnel and many accelerator components.

The time allocated for the underground tour had to be optimised, since we wanted to maximise the number of visitors and give them the opportunity to see and understand as much as possible. After evaluating different scenarios, given the limits imposed by the safety rules, we decided on groups of fourteen visitors accompanied by one guide and one crowd marshal, who would watch the timing and keep the group under control, going down every four minutes. The duration of the visit, from the moment of entering the lift to go down to the moment of coming out of the lift after the visit, was exactly 28 minutes.

All the guides followed a special training, both in the conference room and underground. In addition to safety issues, emphasis was given to the detailed timing of the whole visit (planned number of stops, allocated time per stop) and the content. During the visit the guides were equipped with portable loudspeakers, which were very useful in the noisy environment underground.

There was a pool of guides and crowd marshals, eager to guide groups in French, English and other languages if there was demand (Italian, Spanish, Polish,...). 

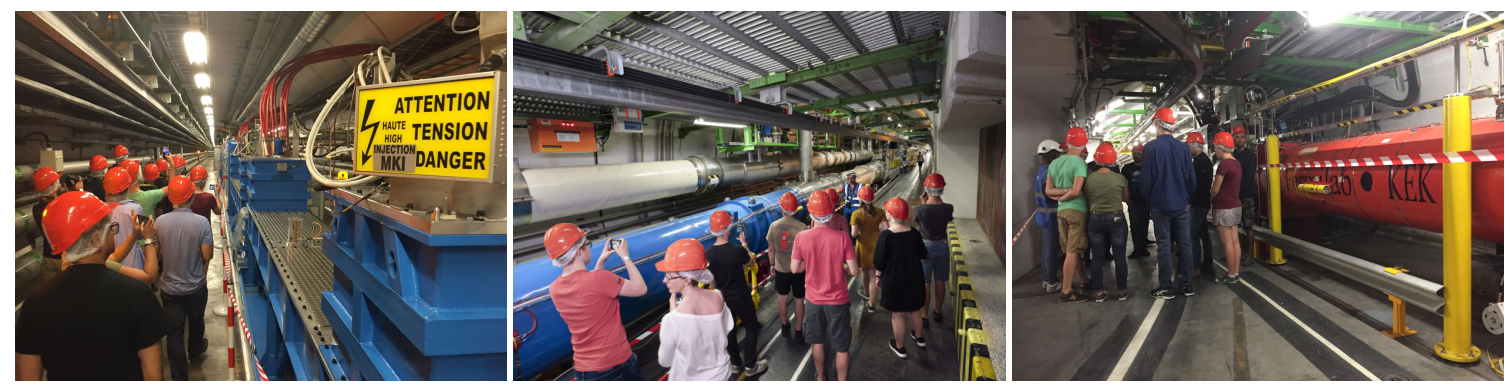

Figure 1: Underground visitors inside the service tunnel (left); inside the straight section of the main tunnel (middle); next to a triplet magnet (right). (Source: CERN)
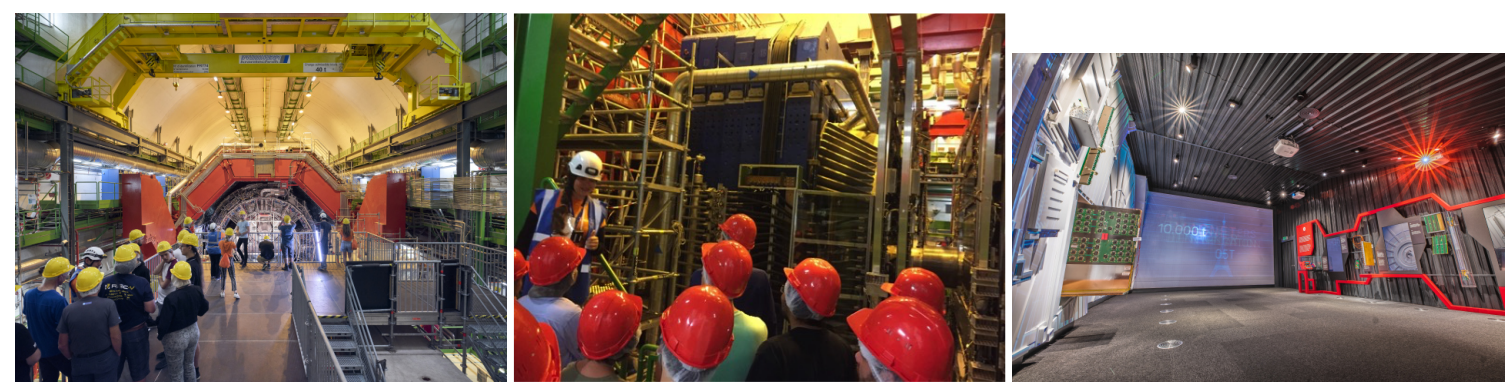

Figure 2: Visitors inside the ALICE cavern, on the low-beta platform in front of the big solenoid (left); next to the forward muon spectrometer (middle). The ALICE exhibition (right). (Source: CERN)

After the distribution of helmets, storing of bags inside cages and a short introduction by the guide while waiting for the lift, the visitors would go down and visit the service tunnel first, where they could see power converters for the LHC magnets and control electronics. Entering the main tunnel at the point where the transfer line from the SPS meets the LHC ring, they could get a glimpse of the arc in the distance and then, inside the straight section, see an LHC dipole, direct feed boxes, kicker magnets and other elements and at the end the triplet for the final focusing of the beams (Figure 1). Inside the ALICE cavern they could admire a front view of the big ALICE solenoid with its huge doors open, half-empty since a major upgrade is happening during LS2; and then a side view of the forward muon spectrometer (Figure 2). Once upstairs, those interested could continue the discussion with the guide and ask questions.

The visitors were encouraged to find out more about ALICE by visiting the ALICE exhibition at the surface (Figure 2). In the exhibition, a seven-minute-long film, which is available in twelve different languages, explains the goals of the experiment, its detectors and the more important results. It is projected on two perpendicular surfaces, one of which is a real-size mock-up of the cross section of the central part of ALICE and with the video mapping technique it brings to life the detector. Some detector pieces are embedded in their actual positions in the mock-up while some more can be seen in show cases, together with a 3D-printed model of ALICE. Three big touch screens offer information about individual detectors. Guides were available inside the exhibition to give further explanations and answer questions after the projection of the film. 


\section{Presentations and "Physics is Fun" show}

In the conference room at Point 2 (building 3294) presentations were given at fixed times, alternating with the "Drôle de Physique" show (experiments with liquid nitrogen). The talks were meant to give the broader picture, not only about ALICE but CERN and particle physics in general, as well as the benefits of our field for society. They were followed by quite some people ( 25 in average, some times up to 40, the full capacity of the room); the audience was of all ages and had many questions for the speakers. The "Drôle de Physique" show was also very popular.

\section{Activities for children and adults}

Inside the big hall at Point 2 (building 2285), all sorts of activities aroused the interest of children and adults and kept them entertained.

A number of experiments, using simple materials, demonstrated various physics principles, such as the magnetic field created by an electric current, gas dynamics (using ping pong balls and a hair dryer), camera obscura and much more (Figure 3). These attracted both children and adults and were a big success.
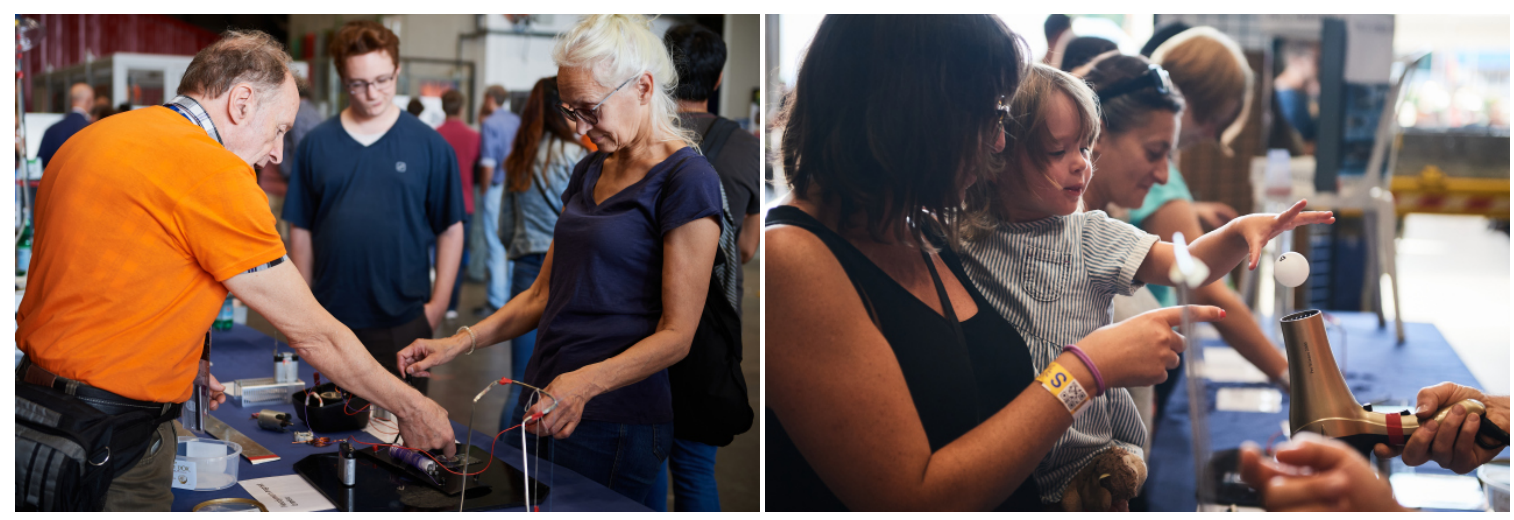

Figure 3: Simple experiments demonstrating physics principles. (Source: CERN)

Right in the middle of the hall, a stand offered children - and their parents - the possibility to build a small, simplified version of ALICE out of Lego blocks. This was part of the LHC Lego, which had been proposed to Lego ideas some years ago [2]. Alternatively, people could build a model of the ALICE detector out of cardboard, by cutting and gluing together its components, parts of the ALICE papercraft [3] (Figure 4). Other games made out of cardboard were on offer: the "fittiscope", introducing to the concept of track reconstruction, which is six wheels with triangles of different colours; these had to be turned so that at least three triangles of the same colour were aligned, defining a particle track; and the particle game - dice with different particles on their six sides, that were thrown and their combination reproduced particle decays.

The cosmic piano [4], demonstrating that we are constantly bombarded by muons, was also on show (Figure 5). This is a set of plastic scintillators with avalanche photodiodes; signals generated by cosmic muons are sent to light bulbs, generating a light flash, and to loudspeakers, generating a sound. Those interested could find out more about cosmic rays and particle detection by discussing with the animators. 

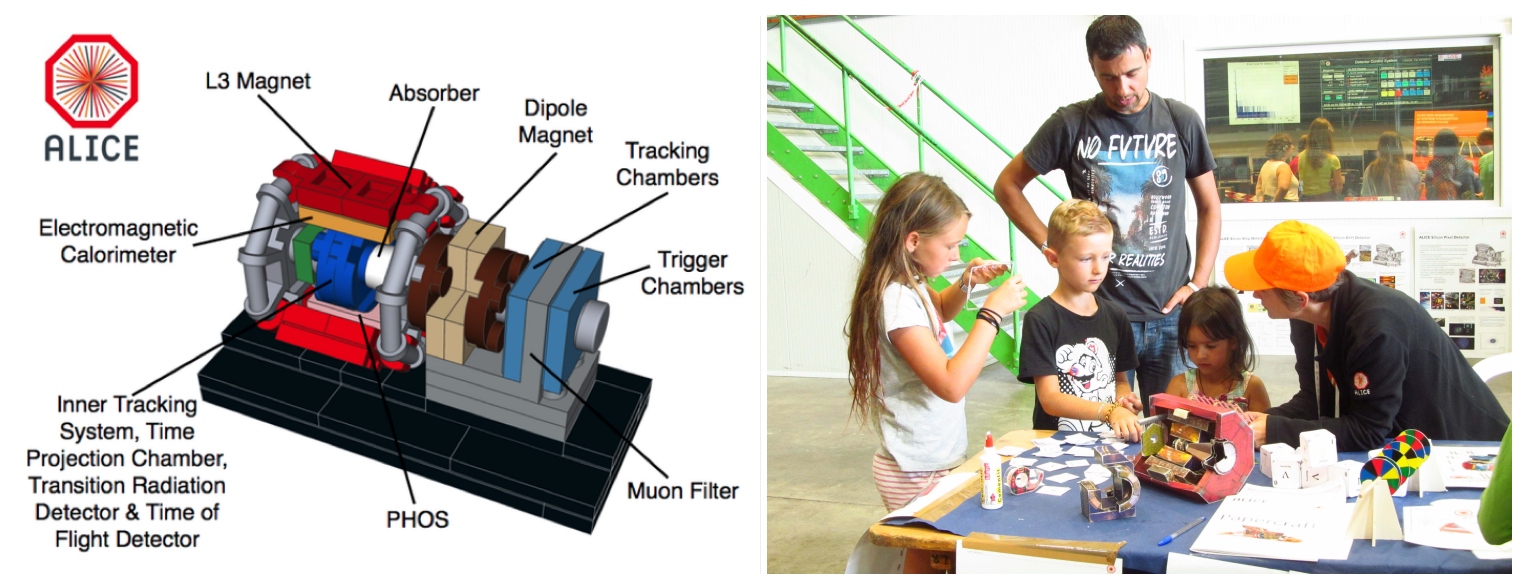

Figure 4: The ALICE detector made of Lego blocks (left); building ALICE out of cardboard, the ALICE papercraft (right). (Source: CERN)

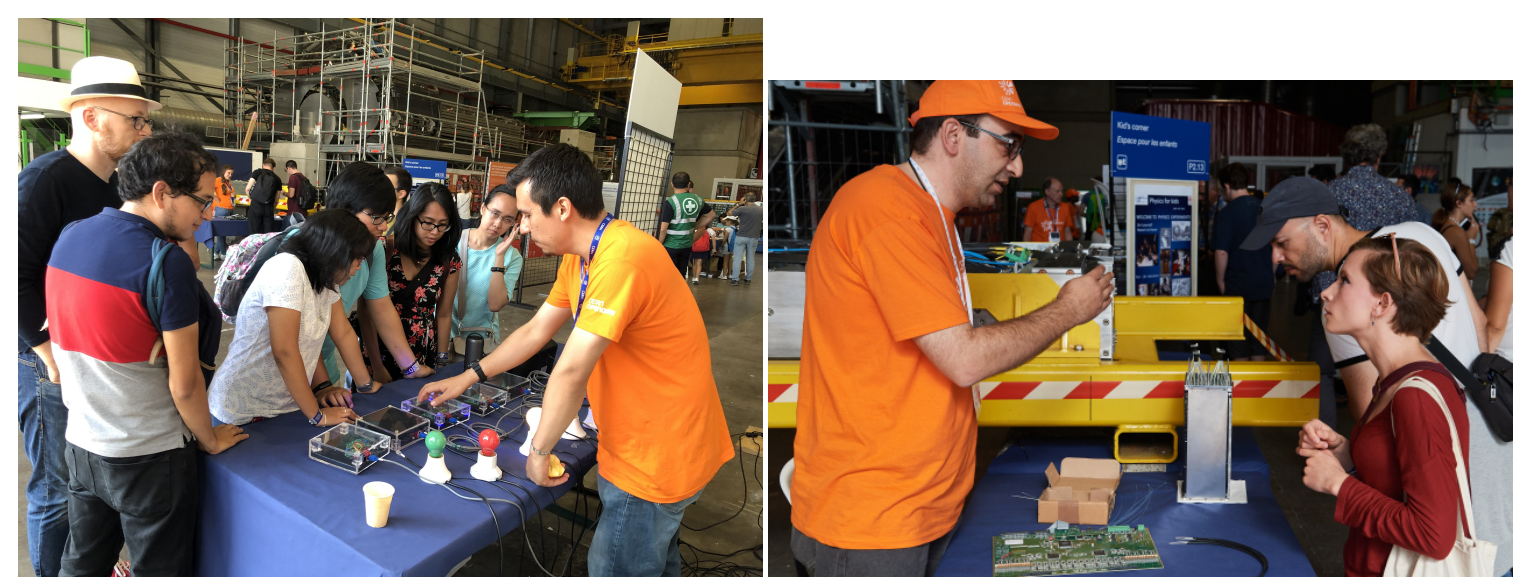

Figure 5: The cosmic piano (left); the electromagnetic calorimeter (right). (Source: CERN)

Stands with detectors inside the hall allowed the visitors to see up close and touch some real detector pieces. A supermodule of the Transition Radiation Detector, which combines tracking and electron identification, was on show, since it had come on the surface for maintenance work on the electronics during LS2. Also a module of the Electromagnetic Calorimeter, again on the surface for maintenance, was on display (Figure 5). Support material - posters and videos - provided additional information, and experts from these detectors were available for explanations.

\section{Inside the ALICE Run Control Centre}

The ALICE Run Control Centre (ARC) had also opened its doors for the visitors. A small DAQ (data acquisition) demo system was on display and DAQ experts were explaining the data taking procedure during Run 3. Virtual reality headsets offered virtual immersion in the LHC tunnel, one of the LHC experiments and the data centre. Also a CAD (Computer Aided Design) - handled, virtual reality environment allowed visitors to virtually explore ALICE (Figure 6). 

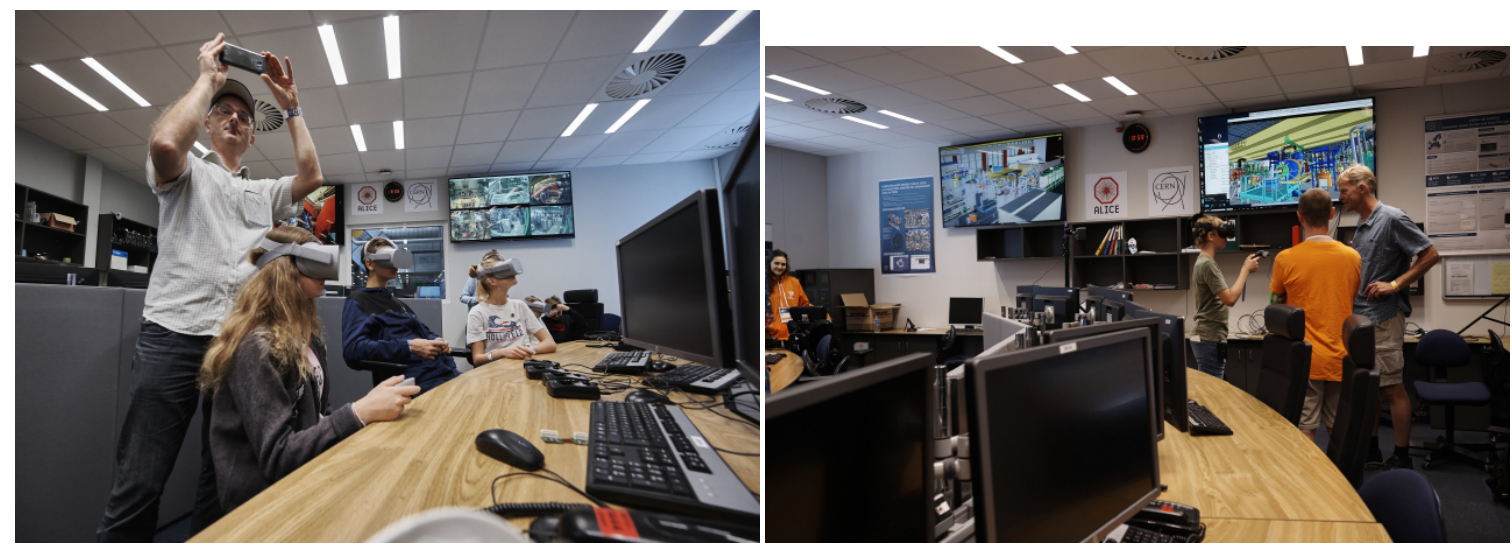

Figure 6: Inside the ARC, virtual reality headsets (left) and CAD-handled virtual reality (right). (Source: CERN)

\section{Summary and Conclusions}

During the CERN Open Days 2019 many thousands visited Point 2. A total of 4600 people went underground; 600 on Friday afternoon, which was dedicated to CERN staff, families and friends, 1800 on Saturday and 2180 on Sunday. In addition to visitors from the CERN area, France and Switzerland, many had come from far away, specifically for the open days. People were happy in general - even though during peak time they had to queue an hour or more for the underground visit - and grateful for the opportunity.

All this was possible thanks to more than 200 volunteers, half of them ALICE members, who acted as guides, lecturers, animators for the activities or helped with logistics and other necessary tasks. Their undiminished enthusiasm, in spite of the long hours, and their eagerness to share their knowledge was clearly appreciated by the visitors.

This event also gave the chance to initiatives of individuals to become appreciated by many.

Hopefully the experience from the Open Days will have a long-term effect, bringing to our colleagues the message that outreach is rewarding and will encourage many to become active. We are looking forward to more events of this type, and to the next Open Days, in some years' time.

\section{Acknowledgements}

We thank Mirko Pojer for enlightening us about the various elements inside the service tunnel and the main tunnel of the LHC.

\section{References}

[1] https://cds.cern.ch/collection/Posters?ln=en

[2] https://ideas.lego.com/projects/94885

[3] http://alicematters.web.cern.ch/?q=PapercraftOpenDays2013

[4] http://alicematters.web.cern.ch/?q=ALICEcosmicpiano 